Questions vives

\section{Questions Vives}

Recherches en éducation

Vol. $9 n^{\circ} 19$ | 2013

Éducation routière, changement de comportement et formation à la conduite : constat, enjeux et transformations

\title{
Formation Tout au Long de la Vie à une conduite sûre : enjeux et limites d'un dispositif d'apprentissage
}

Long Life Learning to safe driving

Marianne Abramovici et Catherine Maman

\section{(2) OpenEdition}

Journals

\section{Édition électronique}

URL : http://journals.openedition.org/questionsvives/1280

DOI : 10.4000/questionsvives. 1280

ISBN : 978-2-8218-1394-6

ISSN : $1775-433 \mathrm{X}$

Éditeur

Université Aix-Marseille (AMU)

Édition imprimée

Date de publication : 15 juillet 2013

Pagination : 89-106

ISBN : 978-2-912643-43-8

ISSN : 1635-4079

Référence électronique

Marianne Abramovici et Catherine Maman, «Formation Tout au Long de la Vie à une conduite sûre : enjeux et limites d'un dispositif d'apprentissage », Questions Vives [En ligne], Vol. $9 n^{\circ} 19$ | 2013, mis en ligne le 15 octobre 2013, consulté le 01 mai 2019. URL : http://journals.openedition.org/ questionsvives/1280; DOI : 10.4000/questionsvives.1280

Ce document a été généré automatiquement le 1 mai 2019.

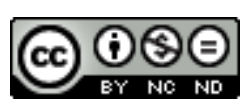

Questions Vives est mis à disposition selon les termes de la licence Creative Commons Attribution Pas d'Utilisation Commerciale - Pas de Modification 4.0 International. 


\section{Formation Tout au Long de la Vie à une conduite sûre : enjeux et limites d'un dispositif d'apprentissage}

Long Life Learning to safe driving

Marianne Abramovici et Catherine Maman

\section{Introduction}

1 Le $1^{\text {er }}$ juillet 2012, a consacré les 20 ans de la disparition du permis de conduire «à vie » au profit d'un " permis à points ». Cette mise en place s'inscrivait alors dans un contexte où l'insécurité routière causait plus de 9000 morts $^{1}$ chaque année.

2 L'objet de cette réforme était de rendre possible, pour les conducteurs infractionnistes, la perte du bénéfice du droit à la conduite, attesté par la possession du permis de conduire, tout en leur offrant la possibilité de récupérer des points en suivant un stage de sensibilisation aux causes et conséquences de l'insécurité routière. Le but était d'amener les stagiaires à changer de comportement sur la route en adoptant une conduite apaisée, respectueuse des autres et des règles.

3 Le permis à points, qui incite le conducteur à avoir une conduite responsable afin de préserver son capital de points, présente donc une double finalité, pédagogique et de prévention.

4 Cette évolution du permis de conduire s'inscrit dans une démarche plus générale, résumée sous le vocable de continuum éducatif dans le domaine de la formation à la Conduite et à la Sécurité Routières (CSR), et repose sur l'idée que l'apprentissage ne doit plus être un point dans le temps (les quelques mois de la préparation du permis de conduire) mais un processus non discontinu qui démarrerait par une sensibilisation dès les premières années de l'enfance pour se poursuivre tout au long de la vie du conducteur. 
C'est ce dispositif de " continuum éducatif » en matière de Formation à la Conduite et à la Sécurité Routières (FCSR) que cet article se propose d'analyser en s'appuyant notamment sur les résultats de deux études portant sur le secteur des écoles de conduite en France (Abramovici, Bancel-Charansol, Jougleux, Maman, 2009). Ces études, réalisées par notre équipe de recherche à la demande de la Direction à la Sécurité et à la Circulation Routière du Ministère des Transports ${ }^{2}$ ont été finalisées respectivement en 2005 et en 2009. La première étude portait sur le repérage des différents acteurs du secteur des écoles de conduite ainsi que sur l'analyse de leur rationalité ; la seconde, a cherché à identifier les conditions d'atteinte des performances (économique, opérationnelle, sociale et sociétale) par les écoles de conduite. Ces recherches actions nous ont amenées à comprendre d'une part, la place des écoles de conduite dans le continuum éducatif et les logiques d'autre part, conduisant les gérants de ces établissements à élargir, ou non, leur offre de formation à d'autres étapes du continuum éducatif.

\section{Encadré méthodologique}

À la demande de l'autorité régulatrice de ce secteur, nous avons conduit en 2003-2005 et en 2006-2009 deux études visant à définir la performance de ce secteur et à en comprendre les leviers. Les performances d'une centaine d'écoles de conduite répartie sur l'ensemble de la France métropolitaine ont été analysées au cours de ces deux études, nous permettant de spécifier précisément l'offre « normale » de formation à la conduite et son processus de production standard. Au cours de ces six années, nous avons pu nous entretenir avec nombre d'experts de ce secteur, soit directement dans le cadre de la phase de cadrage de ces études ou de présentation des résultats (auprès de la délégation interministérielle, d'une commission parlementaire par exemple), soit indirectement, dans le cadre de notre participation à des colloques et journées d'études (notamment dans le cadre du programme PREDIT que nous avons intégré, entre 2005 et 2008). Ces études ont permis d'accéder à une connaissance fine du secteur et d'être en mesure d'identifier aux cours de nos entretiens des pratiques innovantes révélées par les exploitants en contradiction avec certains discours des experts sur l'absence d'innovations dans ce secteur.

Dans l'étude menée entre 2006 et 2009, nous avons élaboré et administré un guide d'entretien semi directif auprès des 77 entreprises qui ont effectivement accepté de participer à l'enquête. Une partie de ce guide portait sur l'offre de service globale et les partenariats noués. Les entretiens ont été enregistrés et reproduits in extenso. Un codage a ensuite rendu possible une analyse qualitative qui a notamment porté sur l'offre en matière de formation sur l'ensemble du continuum éducatif.

Notre propos dans cet article, sera de montrer en quoi ce dispositif de formation continuée à la Sécurité Routière répond (ou pas) à de nouvelles exigences à la fois en démultipliant les étapes constitutives du continuum éducatif de la FCSR, tout en impliquant un plus grand nombre d'acteurs aux rationalités multiples. Le cadre théorique sous-jacent est celui de la sociologie de la traduction proposée par Bruno Latour (1991) et Michel Callon (1986).

7 L'exposé détaillé du contenu et de l'articulation des étapes constitutives du continuum éducatif de la Formation à la Conduite et à la Sécurité Routières, dans une première partie de cet article, sera nécessaire à la compréhension de ses enjeux qui seront présentés dans 
une seconde partie. Ces enjeux seront exposés en nous intéressant successivement d'une part, aux spécificités des compétences à acquérir pour être usager de la route et aux évolutions du contexte sociétal dans lequel s'inscrit cet usage puis, d'autre part, en resituant le continuum éducatif de la Sécurité Routière dans un schéma socioéconomique plus vaste, celui de la Formation Tout au Long de la Vie (FTLV).

\section{Description du continuum éducatif en matière de sécurité routière : Une formation « du berceau au tombeau »}

8 La réflexion sur le continuum éducatif appliqué aux usagers de la route croise deux dimensions: spatiale (une multitude d'acteurs concernés) et temporelle (l'inscription dans la durée et dès le plus jeune âge, d'actions de sensibilisation et d'apprentissage).

Un des résultats de la recherche en éducation routière est la nécessité d'améliorer la formation du conducteur en augmentant, non pas sa formation initiale, mais l'expérience de la conduite dans des conditions de moindre risque, et au travers notamment d'un processus d'autoévaluation de ses comportements sur la route. Il s'agit de s'appuyer sur un processus d'apprentissage basé sur un système d'accès graduel à la conduite, intégrant davantage la formation à la Sécurité Routière, dans le cadre d'une éducation à la sécurité routière progressive et continue tout au long de la vie (du berceau au tombeau, pour reprendre l'expression américaine équivalente). Cette démarche est résumée sous le vocable de continuum éducatif et consiste à agir conjointement sur tous les leviers et déterminants de cette éducation à savoir le temps, l'âge, les acteurs et les méthodes pédagogiques.

10 En effet, pour devenir un conducteur "sûr » la formation doit s'inscrire dans le temps ; on ne peut se former en quelques semaines ou en quelques mois avant le passage d'un examen qui ne sanctionne, comme tout examen, qu'une partie des aptitudes. C'est dès l'enfance que l'usager de la route doit commencer à se former, et il doit continuer à le faire durant toute sa vie. La conduite automobile nécessite un apprentissage spécifique et continu incorporant aussi des éléments d'ordre culturel.

11 Un travail spécifique doit être mené à chaque âge en fonction à la fois des différents statuts d'usagers de la route (piéton, cycliste ....) mais aussi des différents modes d'acquisition des connaissances propres à chaque âge. Le dispositif de continuum éducatif sous-tend une mobilisation générale de tous les acteurs (parents, jeunes, enseignants de l'Éducation nationale, de la conduite, psychologues, travailleurs sociaux, associations, assureurs, entreprises).

12 Les méthodes pédagogiques adaptées favorisent les échanges et les réflexions ainsi que l'autoévaluation à l'égard des risques routiers. Dans ce contexte, le caractère permanent et continu de la formation à la conduite constitue la clé de voûte du dispositif de formation et de validation des connaissances en matière de sécurité routière. Ce continuum éducatif s'est traduit en France par l'affichage d'un processus de formation en quatre étapes correspondant à quatre périodes de la vie d'un conducteur: la période scolaire et périscolaire, la période d'apprentissage anticipée, celle de l'apprentissage au permis définitif, la période de suivi. 
13 Après avoir rapidement rappelé sous quelle forme est actuellement reconnue l'aptitude à conduire une automobile dans le cadre du permis à points, nous présenterons ensuite chacune des étapes du continuum éducatif en décrivant le public visé, les objectifs de la formation et les acteurs impliqués dans la formation à la conduite et à la sécurité routière.

\subsection{Une sensibilisation à la sécurité routière dès l'enfance}

e public visé à la première étape (scolaire et périscolaire) est un usager de la route de moins de 16 ans, donc piéton ou cycliste. L'enfance et l'adolescence sont des périodes propices à l'acquisition de compétences et d'attitudes indispensables aux futurs «bons conducteurs ». Elles sont considérées par les experts comme des temps de "formationinvestissement $»^{3}$ car l'individu est a priori très réceptif aux enseignements sur les dangers de la route et les comportements sûrs à adopter.

Si la famille et, plus largement l'entourage de l'enfant, ont un rôle essentiel à jouer notamment à travers l'exemplarité de leurs comportements sur la route, l'école est le vecteur privilégié de cette éducation précoce à la sécurité routière, le lieu où doit s'effectuer le travail sur l'intériorisation de la loi ainsi que son appropriation. Le programme d'action mis en œuvre par l'Éducation Nationale commence dès la maternelle et se poursuit durant toute la durée de la scolarité obligatoire. À l'issue de la scolarité primaire, l'élève doit avoir acquis les règles et les comportements de sécurité liés à l'usage de la rue et de la route en sécurité, dans sa vie quotidienne (chemin de l'école, déplacement en voiture avec la famille). L'élève doit acquérir des savoirs (règles élémentaires de circulation, de sécurité...), des savoir-faire (capacités à traiter l'information, à anticiper, à mener en parallèle plusieurs actions...), ainsi que des savoirêtre tels que le respect des règles et des autres usagers. Plus généralement, il s'agit pour les jeunes de comprendre les enjeux de la sécurité routière ainsi que les facteurs de risques routiers. Mais au-delà, il s'agit aussi d'apprendre à connaître les tendances en matière de risque, que ce soit dans la vie de tous les jours ou en matière de sécurité routière, car il $\mathrm{y}$ a très souvent des liens entre ces deux derniers points.

16 Si la question de la formation à la Sécurité Routière dans le cadre de l'Éducation Nationale n'est pas nouvelle, elle date de 1957, l'évaluation de cette étape du continuum éducatif a été instauré plus récemment et repose sur trois évaluations formelles : l'Attestation de Première Éducation à la Route à l'école primaire depuis 1993, l'Attestation Scolaire de Sécurité Routière de niveau 1 en classe de cinquième depuis 2002 et l'Attestation Scolaire de Sécurité Routière de niveau 2 en classe de troisième depuis 2002 également. Il est important de relever que ces évaluations scolaires ont trouvé par la suite une valorisation sociale, puisque depuis 2002, l'ASSR $1^{4}$ est nécessaire pour l'obtention du Brevet de Sécurité Routière exigée pour la conduite d'un cyclomoteur et l'ASSR2 est exigée pour l'inscription au permis de conduire.

17 Pour autant, si la formation à la sécurité routière est présente dans les programmes, les moyens fournis pour assurer sa mise en œuvre semblent insuffisants. La formation des enseignants à la Conduite et Sécurité routière n'est pas suffisante (il n'existe pas de module spécifique dans leur formation) et l'investissement des enseignants relève largement du volontariat et de l'autoformation, facilitée, il est vrai pour cette dernière, par l'accès à de nombreux supports pédagogiques en ligne et par la possibilité de s'appuyer sur les délégués départementaux de l'Éducation Nationale. 


\subsection{Conduire avant 16 ans : une question d'accompagnement} possible d'apprendre à conduire une automobile à partir de 16 ans $^{5}$ dans le cadre de l' Apprentissage Anticipé de la Conduite (AAC). En effet, ce dispositif introduit, en complément de la formation théorique et pratique classique, une période de conduite accompagnée d'une durée d'au moins un an et d'au plus trois ans afin de parcourir 3000 kilomètres minimum. Le jeune conducteur bénéficie ainsi, en plus d'une formation classique, « d'une éducation informelle » (Assailly, 2005) visant à lui permettre d'acquérir une expérience de conduite dans un cadre protégé (conditions de conduite limitées, présence de l'accompagnateur). de formation et plus généralement leur relation de service (Abramovici et al., 2009). En effet, c'est l'école de conduite qui doit expliquer l'objectif de la conduite accompagnée aux accompagnateurs, souvent les parents. Trois rendez-vous pédagogiques sont prévus afin à la fois d'évaluer l'expérience acquise par l'apprenti-conducteur (épreuve pratique) et aussi d'animer les échanges entre tous les protagonistes concernés, autour de l'analyse des situations de conduite rencontrées par l'élève.

L'AAC a donc introduit une coproduction de la formation à la conduite en légitimant le rôle des accompagnateurs-parents dans cet apprentissage et en chargeant le prestataire principal de l'encadrement de ces accompagnateurs. Maryse Pervanchon rappelle que dans le projet initial, les conséquences positives d'une formation indirecte, prise en charge par l'enseignant de conduite à destination des accompagnateurs, sont explicitement envisagées (Pervanchon, Bouzigues \& Acourt, 2004). Cependant, outre les réserves concernant la qualité des rendez-vous pédagogiques (Chatenet \& Leroux, 1999), on peut noter que cet argument n'est pas toujours présent dans la promotion institutionnelle du dispositif.

21 Par ailleurs, l'introduction des accompagnateurs dans le dispositif de formation a rendu nécessaire la création (innovante dans ce secteur) d'un outil de gestion de la relation pédagogique sous la forme d'un livret d'apprentissage. Cet outil, conçu pour être prioritairement utilisé et rempli par l'apprenti-conducteur, permet à la fois de formaliser les objectifs et les étapes de sa formation tout en rendant possible, pour tous les partenaires du dispositif, le suivi des apprentissages de l'élève.

À l'issue des 20 heures de formation pratique, l'école de conduite délivre une Attestation de Fin de Formation Initiale qui permet à l'apprenti conducteur de commencer sa période de conduite accompagnée. À la fin de celle-ci, et dès lors que l'évaluation réalisée au cours $\mathrm{du}$ troisième rendez-vous pédagogique par l'école de conduite, a été satisfaisante, le candidat est présenté à l'examen du permis de conduire. L'obtention du permis de conduire, qui est un certificat de capacité, sanctionne alors la reconnaissance d'une aptitude de l'individu à exercer une activité de conduite. Les titulaires du permis de conduire ayant bénéficié de l'AAC doivent respecter une période probatoire plus courte : 2 ans au lieu de 3.

23 Ce dispositif de formation, avec un taux de réussite en première présentation bien supérieur à la formation traditionnelle ( $71,5 \%$ en 2011 soit un écart de 17,4 \% par rapport à la formation initiale $\left.{ }^{6}\right)$, n'a cependant pas tenu ses promesses en matière de sécurité routière (les évaluations montrent l'absence de distinction dans l'implication accidentelle 
entre les bénéficiaires de l'AAC et ceux qui ont suivi une formation traditionnelle). Les spécialistes s'accordent sur la nécessité d'une formation accrue et plus spécifique pour les moniteurs et les parents afin de rendre l'AAC plus efficace (Assailly, 2005, p. 59).

\subsection{Sur la route du permis}

24 Du point de vue comportemental, les besoins d'apprentissage des jeunes entre 18 et 25 ans, correspondant au public de la troisième étape du continuum d'éducation à la sécurité routière, sont spécifiques. D'une part, cette catégorie de demandeurs, notamment les jeunes hommes, adoptent des comportements plus risqués. D'autre part, ils évaluent et acceptent le risque différemment. Aussi les choix éventuels de situations à risque résultent autant d'une incapacité à percevoir le degré de risque attaché à une décision que de la volonté de se placer délibérément dans des situations à risque. Le rapport Verré souligne sur ce sujet que «les besoins en apprentissage portent sur les compétences perceptives et cognitives et les besoins en éducation nécessitent d'intervenir sur les motivations personnelles et le sens des responsabilités sociales ».

Ainsi, pour ceux qui n'ont pas pu ou pas voulu bénéficier de l'AAC, la phase d'apprentissage initiale traditionnelle du permis de conduire à partir de 18 ans est une étape essentielle du cursus continu d'éducation et de formation à la sécurité routière.

L'enseignement à la conduite, dispensé par les écoles de conduite, doit être conforme aux objectifs pédagogiques du Programme National de Formation à la Conduite (PNF) ${ }^{7}$ qui stipule les objectifs d'acquisition des compétences minimales d'un conducteur « sûr » en matière de savoirs, savoir-faire et de savoir-être.

27 Cette formation est organisée en deux étapes : «La formation théorique (ou code) est axée sur l'apprentissage des règles de conduite et des bons comportements en matière de sécurité routière. Elle comprend des leçons théoriques et des tests. La formation pratique est axée sur les bons comportements du conducteur afin de ne mettre en danger ni sa propre sécurité ni celle des autres. » (Présentation officielle, dépliant permis B, site de la sécurité routière).

Depuis 2002, l'épreuve théorique du permis de conduire a été rénovée avec des questionnements plus largement centrés sur les comportements, les risques et la sécurité. De même, l'épreuve pratique (renforcée et rallongée de 22 minutes à 35 minutes) est, depuis 2010, davantage centrée sur un bilan de compétences autour de trois compétences clés : connaître et maîtriser son véhicule, appréhender la route et partager la route avec les autres usagers. Il s'agit ainsi de pallier les limites d'une formation, discontinue et limitée dans le temps, qui se borne trop souvent, non à un apprentissage de la conduite sûre, mais à la préparation de l'obtention du permis de conduire en se concentrant exclusivement sur l'acquisition des règles du code de la route et du maniement d'un véhicule. De nombreux auteurs regrettent que, dans les faits, la formation n'intègre pas suffisamment des niveaux supérieurs du comportement (attitudes au volant et connaissance de soi par rapport à la prise de risque) (Matrice Gadget $\left.{ }^{8}\right)$.

\subsection{Le permis obtenu... la formation continue...}

Depuis le $1^{\text {er }}$ mars 2004, afin de renforcer la responsabilisation des conducteurs novices, ces derniers disposent pendant une période de trois ans d'un permis "fragilisé » comportant six points et non plus 12 comme antérieurement. Pendant leur période 
probatoire 9 , toute année, durant laquelle aucune faute ne donnant lieu à un retrait de point n'est constatée, entraîne une majoration de deux points (trois points pour les AAC), ce qui permet aux conducteurs ayant fait un "parcours sans faute" d'acquérir l'intégralité de leurs points à l'issue de leur période probatoire. Par ailleurs, les conducteurs dont le permis a été annulé ou invalidé sont aussi concernés par le permis probatoire.

Lorsqu'ils perdent trois points ou plus en une fois pendant la période probatoire, les conducteurs doivent suivre un stage de sensibilisation à la sécurité routière de deux jours. Le stage (identique à celui du permis à points) vise à faire réfléchir à sa conduite et à son comportement à l'égard des autres, afin d'éviter la réitération de nouvelles infractions. En cas de perte de six points en une seule fois (pour une conduite en état alcoolique par exemple), le permis est invalidé.

La formation à la conduite et à la sécurité routière, dispensée à l'issue de l'obtention du permis de conduire, constitue la quatrième et dernière étape de continuum éducatif. Elle concerne des publics très vastes, dans des situations et des demandes très variées.

La formation la plus connue est sans doute celle dispensée dans les stages visant à récupérer des points perdus, après une infraction. En effet, le conducteur auquel plus d'un point est retiré peut les récupérer, s'il ne commet pas de nouvelle infraction dans les trois ans qui suivent la sanction. Le conducteur a cependant la possibilité de récupérer des points plus rapidement en suivant un stage spécifique centré sur le comportement du conducteur et la sécurité routière. Ces stages de « reconstitution des points du permis de conduire ", financés par le participant, sont un droit pour les conducteurs infractionnistes.

En 2010, la France a enregistré 10 millions de points retirés, 85000 permis à points annulés et 2,3 millions de permis reconstitués après 3 ans de conduite sans infraction ${ }^{10}$. On peut lire sur les sites officiels « parmi les 37 millions de conducteurs en France, $90 \%$ détiennent au moins 10 points sur leur capital de permis à points, $75 \%$ ont leurs 12 points de permis en $2009^{11}$.

Le Comité Interministériel de la Sécurité Routière (CISR) s'engageait le 13 janvier 2009 dans une nouvelle réforme du permis de conduire en se fixant un triple objectif : une formation au permis de conduire moins longue, moins chère, et plus sûre. Dans ce contexte, la réglementation du permis à points a été revue depuis, à plusieurs reprises. On citera à titre d'illustration, la loi du 16 mars 2011qui réduit le délai de récupération de un point (de un an à 6 mois). Par ailleurs, les textes du Journal officiel publiés le 3 janvier 2012 sanctionnent le visionnage d'un écran au volant qui passe de $135 €$ d'amende et retrait de deux points à $1500 €$ et retrait de trois points, ou encore prohibent la détention de matériel de détection des radars (sanctionnée par $1500 €$ d'amendes et de 6 points de retrait) ${ }^{12}$. Cependant, selon les chiffres de la Sécurité Routière, 3963 personnes ont été tuées sur les routes en 2011, contre 3992 en 2010. L'année 2011 marquerait donc un nouvel essoufflement dans la lutte contre l'insécurité routière, avec une baisse de seulement $0,7 \%$ de la mortalité.

Le contenu et l'approche pédagogique de ces stages ont été fixés par l'administration s'appuyant en particulier sur les travaux de l'Institut National de Recherche sur les Transports et la Sécurité (INRETS). Le stage, d'une durée de 16 heures, comprend un module de tronc commun qui consiste à donner des informations, notamment des statistiques sur les accidents de la route, ainsi que des éléments de connaissance sur les 
comportements du conducteur Des modules spécialisés portant sur la vitesse ou les problèmes liés à l'alcool peuvent aussi être proposés. Ces stages, également proposés par les procureurs de la République dans le cadre de l'alternative aux poursuites judiciaires, recourent à des pratiques pédagogiques, relativement innovantes dans ce secteur, qui reposent sur la co-animation par un psychologue et un moniteur de conduite titulaire du Brevet d'Aptitude à la Formation de Moniteurs (BAFM), ainsi que sur la dynamique de groupe. Il s'agit de faire prendre conscience à chacun de son propre comportement sur la route et des risques associés. Dès lors qu'un participant a suivi le stage avec assiduité et s'est acquitté du coût de sa formation, il obtient la restitution d'un maximum de quatre points perdus (six pendant la période probatoire). Les centres agréés par les préfectures sont souvent en lien avec les écoles de conduite ou des centres de formation d'enseignement. Un grand nombre d'entre eux sont constitués en réseau: Prévention Routière, Association Nationale pour la Promotion de l'Éducation Routière (ANPER), Formation Sécurité Routière, AFT, Promotrans. Notre étude sur le secteur des écoles de conduite a souligné le petit nombre d'écoles de conduite qui intègrent cette formation dans leur offre de service. Seules les structures les plus importantes parviennent à organiser des stages de récupérations de points.

Après avoir décrit de manière détaillée le dispositif de continuum éducatif en sécurité routière, nous allons à présent pouvoir en repérer les enjeux.

\section{La Formation tout au Long de la Vie, un Cadre plus général du continuum éducatif en sécurité routière : l'animation imparfaite d'un réseau}

\subsection{Les enjeux du continuum éducatif en FCSR}

La spécificité des comportements de conduite comme la maîtrise du risque routier nécessitent non pas l'amélioration de la formation initiale du conducteur, mais plutôt une expérience continue de l'usage de la route.

La spécificité des comportements de conduite tient notamment au fait que s'ils sont en apparence individuels (on est seul au volant et seul maître de son véhicule) ils s'inscrivent cependant bien, dans une dimension éminemment collective du fait du partage de la route qui amène à prendre en compte les autres usagers. On relèvera d'ailleurs que si le plus souvent notre recherche s'intéresse aux conducteurs d'automobiles, le continuum éducatif concerne l'ensemble des usagers de la route, à commencer par les piétons, mais aussi, les cyclistes et autres conducteurs de deux-roues, véhicules poids lourds...

Afin de mieux comprendre d'une part, les caractéristiques des comportements de l'usager de la route, et d'autre part, la nécessité d'inscrire la formation de ces comportements dans une démarche progressive et continue que rend possible le continuum éducatif, il est important de rappeler quelles sont les spécificités des compétences nécessaires à la conduite, avant de présenter de manière plus détaillée, une série d'évolutions sociétales ayant influencé les comportements sur la route, en faisant apparaître de nouveaux risques qu'il convient de prendre en compte dans le processus de formation. 


\subsubsection{La spécificité des compétences à acquérir pour être usager de la route} 1997). freiner, débrayer...

Conduire est une activité complexe et la qualité de la compétence maîtrisée en la matière ne tient pas tant au geste en lui-même qu'à « la mise en œuvre de nombreuses facultés dont celles d'attention, d'observation et d'interprétation d'une situation se modifiant rapidement » (Bailet, 1997). Selon D. Schuffenecker « chaque conducteur fait en moyenne 200 observations et prend 20 décisions sur un parcours routier de 1 kilomètre » (Bailet,

41 Trois niveaux de complexité croissante sont retenus dans le rapport Verré pour définir l'activité de conduite: " une tâche de contrôle de trajectoire, une tâche de résolution de situation et une tâche globale de navigation et d'orientation en circulation. ${ }^{13}$

Ainsi, pour conduire, l'individu met en œuvre des compétences à la fois techniques et comportementales, imbriquées les unes aux autres de manière indissociable.

Par compétences techniques, nous entendons des savoir-faire : savoir passer des vitesses,

Les compétences de nature comportementale se définissent quant à elles par des savoirêtre s'exprimant par exemple à travers des comportements civiques, ce qui soulève ici la question de la frontière entre formation et éducation.

En effet, comme nous l'avons vu dans la première partie de cet article, la famille joue un rôle important dans la socialisation des futurs usagers de la route par l'imprégnation, dès la petite enfance, des comportements observés in situ. Au-delà des comportements familiaux et des influences qu'ils produisent, il s'agit bien aussi de comportements culturels plus profonds. Nous avons lors de notre étude rencontré des enseignants de la conduite qui relevaient la récurrence des comportements d'infractions (stops non respectés, feux rouges brûlés...) ou d'incivilités au volant qui étaient le fait d'automobilistes croisés pendant une leçon de conduite. Comment pour un formateur instiller les bonnes pratiques routières alors que l'environnement en fait, de manière répétée, la démonstration inverse?

Car l'apprentissage des bonnes pratiques routières passe avant tout par le mécanisme de socialisation par lequel l'intériorisation des pratiques se fait à travers l'observation puis l'imprégnation répétée des comportements dans son environnement.

\subsection{2. Évolutions sociétales influençant les comportements sur la route}

47 Une fois rappelées quelles sont les spécificités des compétences propres à la conduite, il s'agit à présent de repérer et d'analyser les différentes évolutions sociétales ayant modifié les comportements sur la route.

Vouloir décrire de manière exhaustive l'ensemble des normes, valeurs, croyances, perceptions et modes de représentation contribuant à déterminer de façon directe ou indirecte le double processus de formation à la conduite et de conduite elle-même, serait complexe et renverrait à toute une série de déterminants qui échappent en grande partie au cadre de cet article. Aussi, nous nous sommes contentées de souligner ici l'existence de ces principaux facteurs (au nombre de quatre) en montrant leur évolution récente. 


\section{a. Les représentations du véhicule et le lien à l'altérité} sa conduite en temps réel, il n'a pas encore pour autant reconstruit les liens de complémentarité avec l'ensemble des autres usagers de la route, à savoir les autres automobilistes, les conducteurs de deux-roues et les piétons. À ce propos, une étude de la Sécurité Routière réalisée en 1988 fait apparaître la nécessité de privilégier «le sens collectif » dans la communication sur la Sécurité Routière, en considérant que les comportements de transgression impliqués dans des accidents sont généralement fondés sur un égocentrisme du conducteur. Solidarité et attention portée à l'autre deviennent les vecteurs clés des nouvelles campagnes de communication de la Sécurité Routière.

a lors un tournant dans la nature des slogans publicitaires de la Délégation à la Sécurité Routière qui cherche, à partir du début des années 1990, à insister sur la dimension collective de la conduite routière " ensemble, on est sur la bonne route ». Il n'est d'ailleurs pas anodin de remarquer que l'apparition dès 1976 à l'examen du code, de questions relatives au secourisme, induisait la volonté d'introduire des comportements plus solidaires sur la route. De la même manière, l'Apprentissage Anticipé de la Conduite 
(AAC), ou l'Attestation Scolaire de Sécurité Routière (ASSR) cherchent à affirmer le caractère collectif de la formation à la conduite en impliquant aussi les familles ou l'école dans l'apprentissage à la conduite.

\section{c. La satisfaction de certains besoins à travers des comportements de conduite à risque : besoin d'estime et d'appartenance à un groupe}

Plusieurs études ${ }^{14}$ ont montré que le nombre de jeunes (permis de moins de 3 ans) conducteurs (à dominante masculine) impliqués dans des accidents graves serait particulièrement élevé. Ces résultats renvoient par ailleurs, à la perception du risque chez ces conducteurs, et aussi à la recherche de la satisfaction de deux séries de besoins particulièrement prépondérants dans cette population : le besoin d'estime et de confiance en soi d'une part, et le besoin d'appartenance à un groupe, d'autre part.

On peut montrer que la perception des risques chez les jeunes conducteurs les amène à considérer que les gains liés à une prise de risque sont plus importants que les pertes éventuelles. Ces gains (notamment dans le cas des risques liés à la vitesse excessive) seraient de nature diverse: extériorisation du stress, expression d'un besoin d'indépendance, de prestige au sein d'un groupe, ou encore des raisons pratiques (gain de temps). Le syndrome des jeunes conducteurs regroupe donc des facteurs aussi divers que la confiance en soi, la recherche d'identité, le besoin de se confronter au danger, la recherche de sensations...

Par ailleurs, la conduite sous l'influence de produits tels que l'alcool, les drogues illégales ou les psychotropes est rendue particulièrement dangereuse notamment du fait de l'altération de la perception du risque et de la relation au danger. (On notera que ces facteurs sont de plus en plus mis en avant dans les rapports d'accident.)

Si les normes réglementaires et sociales autour de l'alcool tendent à un rejet partagé de la conduite en état d'ébriété, la prise de conscience des effets négatifs des drogues légales ou non est beaucoup plus récente et ne s'est pas encore traduite jusqu'ici par la même prise de conscience.

\section{d. La conduite : l'école de la vie}

61 L'introduction des dispositifs de conduite accompagnée d'une part, de formations à la sécurité routière dans le cadre scolaire d'autre part, a contribué à souligner le rôle éducatif des parents et des enseignants autour de la sécurité routière. Ce phénomène a participé à la mise en avant des valeurs de respect des règles communes (civisme) et audelà à réintroduire une dimension éthique, voire morale, à la conduite : "apprendre à conduire, c'est apprendre à se conduire» (Pervanchon et al., 2004). Le modèle de l'apprentissage des règles de la vie en société est donc étendu à l'éducation routière. On voit même parfois une inversion de cette analogie, l'apprentissage de la conduite automobile devenant alors l'occasion d'intégrer les règles de vie en société pour un public confronté à des problèmes d'insertion professionnelle ou sociale.

L'ensemble de ces évolutions que nous venons de retracer, combiné à des mesures réglementaires récentes, induit l'émergence de nouveaux acteurs en situation d'apprentissage et donc l'apparition de nouvelles formes d'apprentissage. 

possibles :

- Il peut être mineur et peut soit recevoir une première formation à la sécurité routière dans le cadre scolaire ; soit bénéficier de l'apprentissage anticipé de la conduite en ayant déjà une première expérience des règles de conduite. Ce denier public nécessite un renouvellement des pratiques pédagogiques impliquant les professionnels de la formation à la conduite ainsi que l'environnement familial. Il est à l'origine d'innovations pédagogiques (livret d'apprentissage, rendez-vous pédagogique,...) qui tendent à s'étendre vers d'autres publics.

- L'apprenant à la conduite et à la sécurité peut également être un conducteur confirmé et participer soit à des stages obligatoires prévus dans le cadre du permis probatoire ou du permis à points (conducteur infractionniste), soit à des stages volontaires auxquels il a pu être sensibilisé par les assureurs et/ou l'entreprise dans laquelle il travaille.

Il n'est donc plus possible de caractériser une seule figure de l'apprenant (jeune majeur en situation de primo formation, comme c'était le cas auparavant). Il existe aujourd'hui plusieurs catégories d'apprenants correspondant à des profils, des expériences et des attentes différentes.

Les compétences pédagogiques à mobiliser pour répondre à ces besoins différents sont aujourd'hui multiples. Une partie des nouveaux dispositifs de formation s'appuie d'ailleurs sur la coopération d'acteurs différents (moniteurs et enseignants, moniteurs et parents, moniteurs et psychologues) et nécessitent de ce fait des pratiques de conception collective des formations, totalement innovantes dans ce secteur.

Les évolutions récentes soulignent donc à notre sens les multiples forces qui agissent sur le processus de formation à la conduite et à la sécurité routière. Trois éléments nous semblent importants à souligner :

- d'une part, l'étalement de la formation et la multiplicité des acteurs qu'il entraîne,

- le caractère, d'autre part, progressif de l'éducation routière, ainsi que le processus de mémorisation des acquis que cela suscite,

- et enfin, la diversité des situations de formation à prendre en compte ainsi que leurs implications en termes d'organisation de la formation, de la pédagogie et également de la formation (initiale et continue) des formateurs.

Ce processus ne nous semble pas stabilisé à ce jour, d'où la pertinence, mais aussi la difficulté, à définir ce que devrait être un processus de formation idéale à la conduite et à la sécurité routière dans le cadre d'un continuum éducatif.

\subsection{Des enjeux économiques et sociétaux plus larges : le cadre de la Formation tout au Long de la Vie}

68 Nous allons montrer dans cette partie en quoi le concept de la Formation Continue Tout au Long de la Vie peut enrichir notre compréhension du dispositif de formation progressive et continue à la sécurité routière.

Le concept de Formation Continue Tout au Long de la Vie, traduit de l'anglais Life Long Learning, s'est généralisé à partir de 1996, année désignée par l'Union Européenne comme étant l'année de « l'Éducation et de la formation tout au long de la vie ».

Au-delà de l'ambition originelle portée par la FTLV, garantir une promotion sociale pour tous, deux réalités économiques vont en assurer son développement: la nécessité d'une 
part, de suivre un progrès technique sans cesse plus rapide, et celle d'autre part, de promouvoir la flexibilité des activités économiques en garantissant une mobilité plus importante des acteurs.

Bien que les enjeux soient différents, les principes qui sous-tendent la FTLV, nous semblent, particulièrement bien s'appliquer à la problématique de la Formation à la Conduite et à la Sécurité Routière ; c'est ce que nous allons tâcher de démontrer en développant les deux réalités économiques mentionnées.

\subsubsection{Le rôle du progrès technique}

Force est de constater que le progrès technique pénètre aujourd'hui dans notre quotidien de manière bien plus rapide qu'auparavant. Nous nous approprions à une vitesse déconcertante des objets imprégnés de nouvelles technologies dont nous ne maîtrisons pas toujours spontanément l'ensemble des fonctionnalités. "Ces objets, gérant des processus de plus en plus abstraits, sont de plus en plus opaques.... C'est ainsi que la nécessité d'adaptation aux avancées scientifiques s'impose donc aujourd'hui tout au long de la vie. » (Las Vargnas, 2004).

73 La voiture ne fait pas exception à cette règle, mais, si l'usage par exemple, d'un téléphone portable dont l'intégralité des fonctions est mal maitrisée, représente peu de conséquences en termes de sécurité, il n'en va pas de même pour l'usage de la voiture, dont l'utilisation mal maîtrisée de nouveaux dispositifs associés tels que l'intelligence embarquée, le GPS, le système de freinage automatique, génèrent de nouveaux risques dont il convient de tenir compte dans le continuum éducatif.

74 L'utilisation optimale de ces nouveaux objets ne peut pas se faire exclusivement à l'aide de l'accompagnement institutionnel, on peut se demander dans quelle mesure d'autres acteurs du secteur de la conduite et notamment les constructeurs automobiles ne pourraient pas jouer un rôle dans la formation à l'utilisation de ces nouveaux produits qu'ils commercialisent. Plus généralement, on notera que la mise en place récente par Renault d'une politique de sécurité routière se déclinant par de nombreuses actions qui visent notamment à «faire le choix de système d'information et de prévention, d'équipements permettant au conducteur de concentrer son attention sur l'essentiel, sa conduite (ex. allumage automatique des feux, limiteur de vitesse). Ces systèmes de correction suppléent alors en partie le conducteur. C'est aussi faire le choix de stratégies d'usage qui autorisent toujours le conducteur à prendre la main sur la machine " (site intranet Renault). Même si cette assistance technique au conducteur est aujourd'hui sujette à controverse, il n'en reste pas moins que la démarche du constructeur s'inscrit bien ici, dans un processus de FTLV.

\subsubsection{Flexibilité et mobilité}

75 En parallèle à cette dimension technique, on observe que les investissements aujourd'hui consentis par les entreprises en matière de formation continue répondent de plus au plus au principe de FTLV, et ce d'autant plus que la Loi de mai 2005 instaurant notamment le Droit Individuel à la Formation et le contrat de professionnalisation, est venue renforcer ce dispositif.

76 En effet, dans un environnement économique post-fordiste caractérisé par des évolutions constantes et difficilement prévisibles des attentes des consommateurs, souplesse et 
réactivité des appareils productifs et, par ricochet, de la gestion des Ressources Humaines sont devenus les modes de fonctionnement privilégiés des entreprises. La mise en place des dispositifs de formation à la conduite et à la sécurité routière, aujourd'hui plus contraignants, combinée à la suppression du service national qui offrait la possibilité d'acquérir ces compétences en dehors de la sphère marchande, pose la question de l'accès à la formation comme le traduit l'évolution de la perception du permis par les différentes générations.

Padioleau rappelait que l'obtention du permis de conduire " était une vraie étape, qui marquait la reconnaissance sociale, au même titre, par exemple, que le premier travail. Son obtention allait souvent de pair avec l'acquisition d'une voiture, sorte de rite de passage entre l'adolescence et la vie adulte » (Padioleau, 2004).

En effet, si pour les 30-50 ans le permis était conçu comme « un droit aisément accessible, ne nécessitant pas d'aptitudes ni de moyens particuliers » les plus jeunes le perçoivent "comme un droit à la mobilité, éloigné de la notion de compétence liée à un apprentissage [qui] doit être conquis le plus vite possible $»^{15}$. Ce «droit à la mobilité " traduit bien la perception de l'importance du permis pour réussir une démarche de recherche d'emploi. L'obtention du permis de conduire et sa conservation (dans le cadre $\mathrm{du}$ dispositif de permis à points) sont devenues des composantes essentielles de l'employabilité des salariés).

La politique répressive de contrôle/sanction mise en place à partir de 2003 a exacerbé ces questions en multipliant les contrôles et les retraits de points. La mise en place du permis à points a ainsi créé pour les conducteurs, une nouvelle menace : celle de disposer d'un permis ne leur donnant plus un accès définitif au droit de conduire. Comment dès lors continuer à conserver son activité quand celle-ci nécessite des déplacements en voiture quotidiens? À titre d'exemple, on relèvera le cas - qui nous a été rapporté- de ces délégués médicaux ayant subi un retrait de permis de plusieurs mois, qui n'hésitaient pas à payer quelqu'un pour les véhiculer pendant la période de leur retrait de permis, afin de conserver leur activité, et ce dans la plus grande opacité vis-à-vis de leur employeur. On relèvera à ce propos que si la loi ne fait pas obligation d'informer son employeur d'un retrait de permis, les contrats de travail des salariés itinérants stipulent le plus souvent l'obligation pour le salarié de disposer d'un permis valide.

Ce détour par le cadre général de la FTLV nous permet de montrer qu'au-delà du nécessaire entretien des compétences nécessaires à la sécurité routière, le continuum éducatif en matière de sécurité routière, permet le maintien du droit à la mobilité notamment à travers l'insertion de l'individu dans ses activités économiques et professionnelles.

\subsubsection{L'entreprise : nouvel acteur du continuum éducatif en sécurité routière}

81 Les conducteurs peuvent aussi bénéficier de formation après leur permis de conduire dans le cadre de leur formation professionnelle ; financement de la formation et choix du prestataire sont alors assurés par l'employeur. En 1999, l'État met en place une politique de prévention du risque routier qui s'est traduit par la création d'une Charte de Travail entre la Caisse Nationale d'Assurances Maladie (CNAM), la Direction des risques professionnels et la Direction à la Sécurité et Circulation Routière (DSCR). En 2003, parmi les 5731 morts à la suite d'un accident de la route, il y en avait 810 qui s'étaient produits dans un contexte de travail. Les accidents routiers du travail représentent environ $3 \%^{16}$ 
des accidents du travail toutes causes confondues, et plus de $20 \%$ de l'ensemble des accidents mortels de travail (pour le régime général de la Sécurité Sociale).

Cette politique de prévention du risque routier s'appuie, par ailleurs, sur la signature de chartes de partenariat avec les grandes entreprises nationales ou les fédérations professionnelles. Les entreprises s'y engagent à développer des actions en termes d'évitement du risque (limitation des déplacements, organisation du travail permettant de planifier des durées de déplacement convenables, ...), et d'utilisation de véhicules adaptés et bien équipés (maintenance du parc, formation des salariés, intégration dans la communication externe de la sécurité routière...). Les actions prévues dans ces chartes concernent aussi bien les déplacements professionnels que les déplacements entre le domicile et le travail.

Au-delà de la diminution des accidents de la route, les entreprises espèrent y gagner une réduction des coûts directs et indirects associés à ces accidents ainsi qu'une amélioration de leur image. Les chartes ont pour objectif de favoriser. La mise en place d'un plan de prévention des risques professionnels, intégrant le risque routier dans les entreprises, a conduit celles-ci à faire appel à un réseau de prestataires spécialisés, qui interviennent à la fois dans la formation à la conduite des salariés (les Centres Centaure, Belletoise Evolution, ou encore Prévention Routière Formation) proposent des stages de conduite sur site dédié, de 1 à 2 jours, avec des mises en situation (freinage d'urgence, test des ballons...), d'autres des stages de formation à la sécurité et à la conduite (Prévention Routière Formation) et aussi dans le diagnostic, le conseil et l'élaboration de ces plans de prévention en entreprise.

Un certain nombre de ces prestataires de services se sont rassemblés au sein du GP2R (Groupement des Préventeurs du Risque Routier) au niveau national et ont formalisé des accords et des conventions avec la Caisse Régionale d'Assurance Maladie (CRAM) Ile de France. On y trouve notamment les centres Centaure, Belletoise Evolution Conduire Juste, Prévention Routière Formation, Objectif Maîtrise.

Conçues à titre expérimental en 1999 par la Sécurité Routière en liaison avec les professionnels de l'assurance, des formations de perfectionnement à la conduite sont destinées aux conducteurs novices (Rendez-Vous d'Évaluation) et aux conducteurs expérimentés (Rendez Vous de Perfectionnement, RVP). Les RVE s'inscrivent dans une démarche préventive qui vise à réduire le sur-risque d'accident des jeunes conducteurs. Ce dispositif est conçu pour renforcer la formation initiale par une approche thématique par exemple sur les limites des aptitudes du conducteur, ses différences perceptives mais aussi sur les notions de risque et de responsabilité. Les RVP répondent davantage à une logique d'actualisation des connaissances générales sur la signalisation, l'infrastructure, les règles de circulation et permettent de favoriser la prise de conscience des facteurs de risque (vitesse, alcool, fatigue...).

\section{Conclusion}

En France, la mise en place du continuum éducatif pour la FCSR a permis en 20 ans un accès progressif à la conduite tout en faisant évoluer la figure de l'apprenant, qui n'est plus aujourd'hui seulement celle d'un jeune majeur en situation de primo formation. Plusieurs catégories d'apprenants, correspondant à des profils, des expériences et des 
attentes différentes, se retrouvent confrontées à des modes d'acquisitions de connaissances et de compétences multiples.

Nous avons souligné dans cet article les enjeux et les faiblesses du " continuum éducatif » notamment en ce qui concerne les dispositifs de formation post permis. Notamment, un nombre de points sur un permis ne renseigne ni sur les circonstances des retraits ni sur le niveau du conducteur. Or, il importe de réfléchir à d'autres dispositifs de capitalisation des connaissances sur la conduite d'un individu. Car c'est bien à partir de la mémorisation des comportements que le "continuum éducatif » pourrait prendre tout son sens en permettant aux acteurs de la formation d'agir sur des comportements ciblés et antérieurement repérés.

En inscrivant le dispositif de "continuum éducatif » de la Formation à la Conduite et Sécurité Routières dans le cadre plus général de la Formation Tout au Long de la Vie, l'article a cherché à insister sur l'importance de l'animation d'un réseau complexe constitué d'une multitude d'acteurs souffrant eux-mêmes, pour certains, d'un déficit de formation. Si ce réseau existe, son animation et la coordination des acteurs qui le constituent pourraient être améliorées. En effet, l'articulation des étapes de ce continuum a été correctement pensée du point de vue des formés, elle reste par contre, encore à parfaire du point de vue des formateurs. Les dispositifs qui pourraient permettre à ceuxci d'échanger sur leurs pratiques, leurs difficultés, leurs perceptions respectives des évolutions des connaissances et des savoir-faire, à inscrire dans le programme de formation, restent peu développés. La formation continue des formateurs, qui pourrait constituer un puissant relai de ces réflexions est notoirement insuffisante dans ce secteur. Il nous semble de ce fait important de réfléchir aux formes possibles et souhaitables que cette articulation pourrait prendre. Une analyse comparative entre secteurs d'activité (notamment dans le domaine de l'assurance, de l'automobile, de la formation,...) des différents dispositifs sur lesquels s'appuient les "experts" de la sécurité routière pour bâtir et maintenir leur expertise pourrait constituer un bon point de départ. Dans ce contexte le rôle du délégué à la sécurité Routière pourrait davantage être mis en avant.

\section{BIBLIOGRAPHIE}

Abramovici, M, Bancel-Charansol, L., Jougleux, M., \& Maman, C. (2009). Analyse des performances des écoles de conduite et outils de pilotage du secteur : Rapport final, Laboratoire Organisation et Efficacité de la Production (O.E.P), Université Paris-Est Marne-La-Vallée, Direction de la Sécurité et de la Circulation Routière (D.S.C.R.), Ministère de l'Équipement, des Transports, de l'Aménagement du Territoire, du Tourisme et de la Mer (M.E.D.A.D.D. : Ministère de l'Écologie, du Développement et de l'Aménagement Durable), 126 pages, Octobre 2009.

Assailly, J.-P. (2005). Revue de questions sur le continuum éducatif. Rapport final convention INSERR INRETS. Disponible en ligne : http://www.inserr.org/index.php/la-securite-routiere?start=16

Bailet, J-M. (1997). La conduite routière. Paris :PUF. 
Bertrand J-P. (2005). Faciliter l'accès au permis des Jeunes. Rapport parlementaire 25 Janvier 2005.

Chatenet, F. \& Leroux, P. (1999). Évaluation qualitative d'un mode de formation : l'apprentissage anticipé de la conduite. Rapport de la convention DSCR-INRETS.

Callon, M. (1986). Élément pour une sociologie de la traduction, la domestication des coquilles Saint-Jacques et des marins pêcheurs dans la baie de Saint-Brieuc. L'année sociologique, 36. Paris : PUF.

INSERR, Guide de l'AAC, Novembre 2003

Ministère de l'intérieur (2010). Bilan du permis à points. Direction de la modernisation et de l'action territoriale sous-direction de la circulation et de la sécurité routières, bureau de la sécurité et de la règlementation routières. Disponible en ligne : http://www.interieur.gouv.fr/ Publications/Statistiques/Securite-routiere/Bilan-du-permis-a-points/Bilan-2010

Ministère de l'intérieur (2011). Bilan du permis à points. Direction de la modernisation et de l'action territoriale sous-direction de la circulation et de la sécurité routières, bureau de la sécurité et de la règlementation routières. Disponible en ligne : http://www.interieur.gouv.fr/ Publications/Statistiques/Securite-routiere/Bilan-du-permis-a-points/Bilan-2011

Latour, B. (1991). Aramis ou l'amour des techniques. Paris : La Découverte.

Las Vargnas, O. (2004). La Formation Tout au Long de la Vie : une révolution culturelle qui s'ignore. Colloque apprendre autrement aujourd'hui, Cité des Sciences, Paris 2004.

Padioleau, G. (2004). La réforme du permis de conduire. Le Journal du Dimanche, 29 février 2004.

Pervanchon, M., Bouzigues, M., \& Acourt, G. (2004). Acteurs de la sécurité routière. Actes du séminaire du PREDIT, 9 Avril 2004

Vérré, J. (1997). Table ronde sur la formation des usagers de la route et sur la formation des conducteurs. La Documentation française, Collection : Collection des rapports officiels. Disponible en ligne : http://www.ladocumentationfrancaise.fr/rapports-publics/974072151/index.shtml

\section{NOTES}

1. http://www.interieur.gouv.fr/Publications/Statistiques/Securite-routiere/Bilan-du-permis-apoints/Bilan-2010

2. Actuel Ministère de l'Écologie du Développement Durable des Transports et du Logement

3. Rapport Vérré (Vérré, 1997).

4. ASSR 1 et 2 : Attestation Scolaire de Sécurité Routière

5. Depuis 2002, il faut être titulaire de l'ASSR 2.

6. Bilan des examens du permis de conduire, année 2011, Ministère de l'intérieur, de l'outre-mer, des collectivités territoriales et de l'immigration.

7. Arrêté du 23 janvier 1989.

8. Voir Assailly, 2005.

9. 3 ans pour la formation traditionnelle, 2 ans pour la conduite accompagnée

10. Voir: http://www.interieur.gouv.fr/Publications/Statistiques/Securite-routiere/Bilan-dupermis-a-points/Bilan-2010.

11. Voir : http://www.permisapoints.fr/.

12. Voir : http://www.permis.com/page/permis-a-point_47.

13. Rapport Vérré (1997).

14. Notamment les rapports Vérré et Assailly. 
15. Voir le Rapport Bertrand, 2005.

16. Voir le Dossier Risques routiers Conduire pour son travail, un métier à haut risque, rapport 2012.

\section{RÉSUMÉS}

En inscrivant le dispositif de « continuum éducatif » de la Formation à la Conduite et à la Sécurité Routières dans celui, plus général, de Formation Tout au Long de la Vie, cet article cherche à montrer l'importance du caractère graduel et continue de l'accès aux compétences nécessaires à l'acquisition d'une conduite sûre. Si en 20 ans des progrès ont été constatés en matière de FCSR, la diversité des compétences et des enjeux à l'œuvre, dans la formation à la conduite et à la sécurité routières, justifie le besoin de renforcer la formation des formateurs mais également l'animation et la coordination du réseau d'acteurs contribuant à cette formation.

In this paper we consider the training to driving and Road Security in the more general context of Long Life Learning (LLL). This work focuses on the importance of gradual and continuous access to the skills necessary for the acquisition of safe driving. During 20 years, progress have been made in terms of Road Security, nevertheless, the topic justifies the need to strengthen the training of trainers but also in order to develop LLL, the facilitation and coordination of the network of actors contributing to this training.

\section{INDEX}

Mots-clés : formation, sécurité routière, continu, compétences, conduite Keywords : training, road security, continuous acces, skills, driving

\section{AUTEURS}

\section{MARIANNE ABRAMOVICI}

Institut de Recherche en Gestion, Université Paris Est Marne la Vallée

\section{CATHERINE MAMAN}

Institut de Recherche en Gestion, Université Paris Est Marne la Vallée 